\title{
Smiley Prize
}

The Smiley Prize, established in honour of Donald V. Smiley (19211990), internationally renowned scholar of Canadian politics and a former President of the Canadian Political Science Association, will be awarded in 1998. The prize, to which is attached a monetary award in the amount of $\$ 750$, is awarded biennially to the author or authors of the best book published in English or French in a field relating to the study of government and politics in Canada in the preceding two-year period. The award-winning book may be single-authored or multiauthored and will be selected by the Board of Directors of the Canadian Political Science Association, upon the recommendation of the Prize Jury. No textbooks, edited texts or collections of essays will be considered. The Smiley Prize is sponsored by McGraw-Hill Ryerson Publishers. Individuals who have published a book in 1996 or 1997 which is eligible for the prize should ensure that their publishers forward one copy of the book by December 10, 1997, to each of: (1) Smiley Prize Jury, Canadian Political Science Association, Suite 205, 1 Stewart Street, Ottawa, Ontario K1N 6H7; (2) Smiley Prize Jury, Professor Ronald Manzer, Division of Social Sciences, University of Toronto at Scarborough, 1265 Military Trail, Toronto, Ontario M1C 1A4; (3) Smiley Prize Jury, Professor Thérèse Arseneau, Department of Political Science, St. Mary's University, Halifax, Nova Scotia B3H 3C3; and (4) Smiley Prize Jury, Professor Alain-G. Gagnon, Department of Political Science, McGill University, 855 Sherbrooke Street West, Montreal, Quebec H3A 2 T7.

\section{Prix Smiley}

Le Prix Smiley a été créé en hommage à Donald V. Smiley (1921-1990), grand spécialiste de la politique canadienne dont la réputation dépassait nos frontières et un ancien président de l'Association canadienne de science politique. L'Association décernera le Prix Smiley, d'une valeur de 750 \$, en 1998 à l'auteur, l'auteure ou aux auteurs du meilleur livre publié en français ou en anglais sur un sujet traitant de la politique ou du gouvernement au Canada au cours de la période correspondante de deux ans. Le Conseil d'administration de l'Association désignera le lauréat, la lauréate ou les lauréats à la suite d'une recommandation du Jury du Prix Smiley. Les manuels scolaires, les livres publiés sous la direction d'une personne ou d'une équipe et les collections d'essais ne seront pas admissibles. Le Prix Smiley est subventionné à tous les deux ans par les éditeurs McGraw-Hill Ryerson. Les personnes qui auraient publié un ouvrage en 1996 ou en 1997 selon les conditions du concours devront, pour être éligibles, demander à leurs éditeurs de faire parvenir 